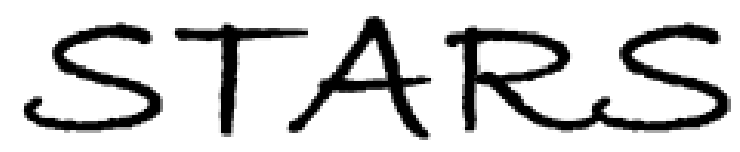

University of Central Florida

STARS

$1-1-2005$

\title{
Super-resolution variable fiber optic attenuator instrument using digital micromirror device (DMD (TM))
}

\author{
Nabeel A. Riza \\ University of Central Florida \\ Farzan N. Ghauri \\ University of Central Florida
}

Find similar works at: https://stars.library.ucf.edu/facultybib2000 University of Central Florida Libraries http://library.ucf.edu

This Article is brought to you for free and open access by the Faculty Bibliography at STARS. It has been accepted for inclusion in Faculty Bibliography 2000 s by an authorized administrator of STARS. For more information, please contact STARS@ucf.edu.

\section{Recommended Citation}

Riza, Nabeel A. and Ghauri, Farzan N., "Super-resolution variable fiber optic attenuator instrument using digital micromirror device (DMD (TM))" (2005). Faculty Bibliography 2000s. 5603.

https://stars.library.ucf.edu/facultybib2000/5603

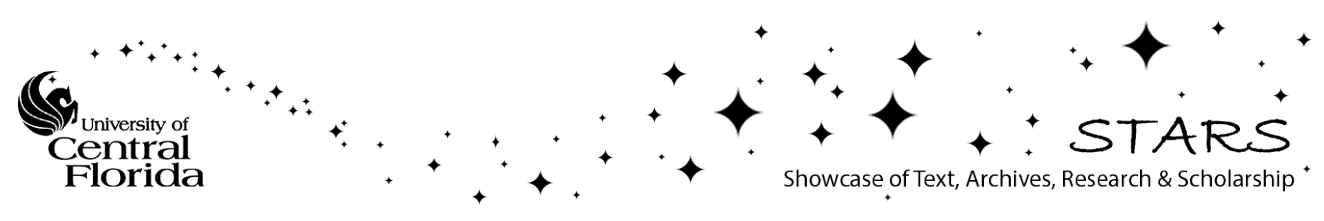




\title{
Super-resolution variable fiber optic attenuator instrument using digital micromirror device (DMDTM)
}

\author{
Nabeel A. Riza ${ }^{a}$ and Farzan N. Ghauri \\ Photonic Information Processing Systems Laboratory, The College of Optics/Center for Research \\ and Education in Optics and Lasers (CREOL), University of Central Florida, \\ 4000 Central Florida Boulevard, Orlando, Florida 32816-2700
}

(Received 18 March 2005; accepted 26 July 2005; published online 2 September 2005)

\begin{abstract}
Demonstrated is a super resolution, low loss, $100 \%$ repeatable and fast response digital variable fiber-optic attenuator instrument using a digital micromirror device. The attenuator design exploits beam expansion optics and a retroreflective architecture to achieve super-resolution with digital repeatability. The proof-of-concept attenuator at $1550 \mathrm{~nm}$ demonstrates a $41.5 \mathrm{~dB}$ optical attenuation range, optical insertion loss of $3.8 \mathrm{~dB}$, a 1 ms maximum attenuation reset time, an optical polarization dependant loss of less than $0.07 \mathrm{~dB}$, and an optical resolution range of $2.9-0.0517 \mathrm{mdB}$. The demonstrated attenuator is appropriate for fiber-optic test and instrumentation applications. () 2005 American Institute of Physics. [DOI: 10.1063/1.2038587]
\end{abstract}

\section{INTRODUCTION}

With the explosion of various optical fiber applications in science, industry, and medicine, there is a need to realize an optical attenuator test instrument for interacting with these single-mode fiber (SMF) systems. This variable fiberoptic attenuator (VOA) fiber-optic test module must be completely repeatable and possess extraordinary capabilities of high resolution attenuation control. In addition, the module should be attractive in terms of speed, optical loss, laboratory conditions environmental reliability, optical operation bands, polarization dependent loss (PDL), and high power optical handling. Recently, several technologies have been proposed for single fiber in-single fiber out VOAs that belong to the all-analog family of VOAs. Example VOA designs include the use of micromechanics, ${ }^{1}$ micromirrors, ${ }^{2,3}$ liquid crystals,${ }^{4-8}$ magneto-optics, ${ }^{9}$ thin-film filters, ${ }^{10}$ long period fiber gratings, ${ }^{11}$ and acousto-optics. ${ }^{12}$ Accurate high resolution operation of analog design VOAs requires closed loop operations of VOA with constant updated module calibration. Recently, an all-digital paradigm was introduced to realize fiber-optic VOAs. ${ }^{13}$ The strength of the all-digital paradigm is its attenuation control through $100 \%$ digital repeatability. Earlier, this digital paradigm has been implemented using the Texas Instruments (TI) digital micromirror device $\left(\mathrm{DMD}^{\mathrm{TM}}\right)$ to realize equalizers ${ }^{14,15}$ and VOAs. ${ }^{16} \mathrm{In}$ this article, shown are the $\mathrm{DMD}^{\mathrm{TM}}$-based VOA module design and its operating characteristics suited to realize a super resolution attenuator for test instrument applications. The rest of the article describes the module design, experimental demonstration, and VOA qualitative performance.

\section{SUPER-FINE RESOLUTION VOA MODULE DESIGN}

Figure 1(a) shows the design of the proposed super resolution $\mathrm{DMD}^{\mathrm{TM}}$ based reflective VOA. The attenuator works

\footnotetext{
${ }^{a)}$ Electronic mail: nriza@mail.ucf.edu
}

on the principles of an in-line self imaging retroreflective design. Input light enters via a SMF and optical circulator $C$ to enter a special self-imaging type fiber lens FL. ${ }^{17}$ Optical circulator is a nonreciprocal device that redirect light from port-to-port sequentially in only one direction, e.g., in a three-port circulator light can be directed from port 1 to port 2 and from port 2 to port 3 . The beam exiting the graded index (GRIN) fiber lens exhibits a specific distance called the half self-imaging distance " $d_{L}$ " over which the Gaussian beam profile exhibits a minimum beam waist. The Gaussian beam exiting the GRIN lens undergoes beam expansion produced by spherical lenses $S_{1}$ and $S_{2}$ with focal lengths $f_{1}$ and $f_{2}$, respectively. The expanded Gaussian beam falls on the surface of a two-dimensional (2D) $\mathrm{DMD}^{\mathrm{TM}}$ populated with many binary state micromirrors [see Fig. 1(b)]. For the attenuator's minimal attenuation setting, all micromirrors in the $\mathrm{DMD}^{\mathrm{TM}}$ are set to generate a retroreflected beam so that maximum input light power is returned via the in-line optics and circulator to the exit SMF. For a given attenuator setting for the VOA, some of the micromirrors are set to an alternate digital state that prevents a certain amount of light from retroreflecting into the output SMF. Thus, a given digital repeatable VOA attenuation setting is achieved by controlling the binary states of the near million micromirrors in the DMD ${ }^{\mathrm{TM}}$.

The positioning of the spherical lenses $S_{1}$ and $S_{2}$, as shown in Fig. 1(a), are carefully picked so as to form an imaging system with beam expansion between the fiber lens minimum beam waist location and the plane of the $\mathrm{DMD}^{\mathrm{TM}}$. Because of this design, a self-imaging SMF coupling condition is satisfied leading to a low loss attenuator design. In addition, the required Gaussian beam waist magnification is enabled to provide a large beam area on the $\mathrm{DMD}^{\mathrm{TM}}$ that leads to the super-resolution capability of the proposed VOA. Specifically, the Gaussian beam expansion can be calculated using ABCD matrix Gaussian optics. One starts with the minimum beam waist radius $\omega_{1}$ at distance $d_{L}$ from the GRIN lens. ABCD matrices laws are used in order to deter- 


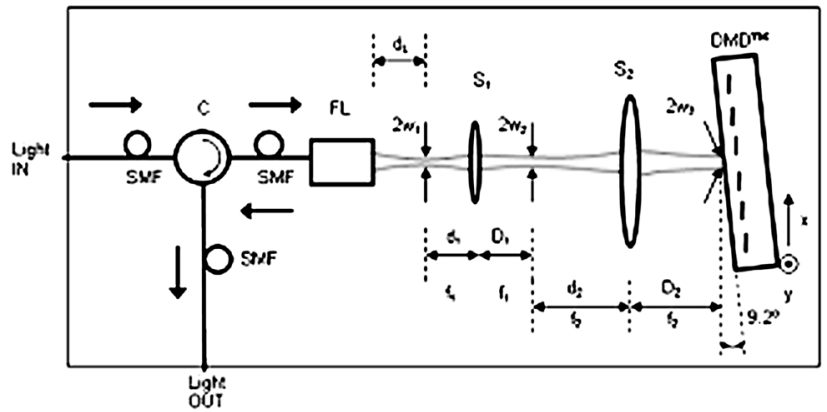

(a)

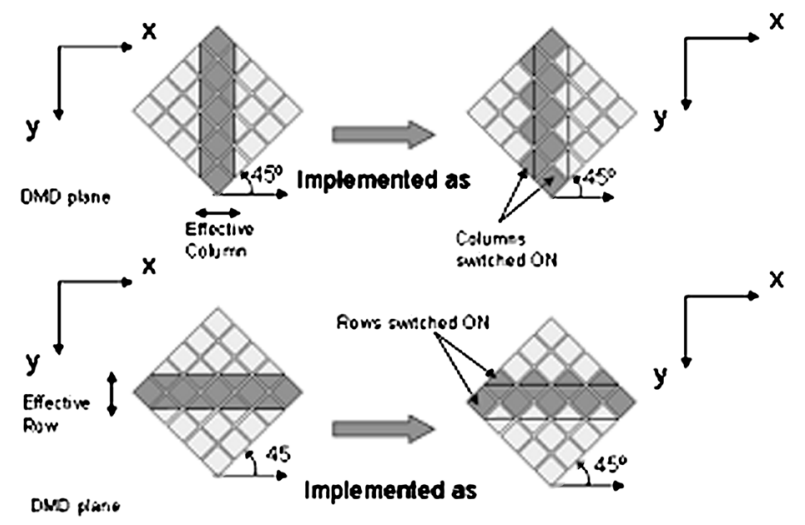

(b)

FIG. 1. (a) Proposed and demonstrated super-resolution VOA instrument using a 2D digital micromirror device $\left(\mathrm{DMD}^{\mathrm{TM}}\right)$. $C$ : circulator, SMF: single mode fiber, FL: fiber lens, $S_{n}$ : spherical lens $(n=1,2), f_{n}$ : focal length of lens $S_{n}(n=1,2), \omega_{n}$ : minimum beam waist before lens $S_{n}(n=1,2,3), d_{L}$ : half self-imaging distance of fiber lens, $d_{n}$ : minimum beam waist distance before lens $S_{n}(n=1,2), D_{n}$ : minimum beam waist distance after lens $S_{n}(n=1,2)$. (b) Figure showing effective column width and effective row width on $\mathrm{DMD}^{\mathrm{TM}}$ for knife-edge experiments performed in the $x$ and $y$ directions, respectively. The figure also shows the actual implementation via individual micromirror control.

mine the state of the Gaussian beam profile after it passes through the lenses. If $d_{n}$ is the distance of Gaussian beam minimum waist $\omega_{n}$ before nth lens and $D_{n}$ is the distance of Gaussian beam minimum waist radius $\omega_{n+1}$ after passing through the spherical lens $S_{n}$, then the following equations describe the beam waists relationships for spherical lenses $S_{1}$ and $S_{2}$ assuming $n=1$ and $n=2$ for $S_{1}$ and $S_{2}$, respectively ${ }^{18}$

$$
\begin{aligned}
& D_{1}=f_{1}+\left(d_{1}-f_{1}\right) \frac{\omega_{2}^{2}}{\omega_{1}^{2}}, \\
& D_{2}=f_{2}+\left(d_{2}-f_{2}\right) \frac{\omega_{3}^{2}}{\omega_{2}^{2}}, \\
& \frac{\omega_{2}^{2}}{\omega_{1}^{2}}=\frac{f_{1}^{2}}{\left(d_{1}-f_{1}\right)^{2}+z_{01}^{2}} ; \quad z_{01}=\frac{\pi \omega_{1}^{2}}{\lambda_{0}}, \\
& \frac{\omega_{3}^{2}}{\omega_{2}^{2}}=\frac{f_{2}^{2}}{\left(d_{2}-f_{2}\right)^{2}+z_{02}^{2}} ; \quad z_{02}=\frac{\pi \omega_{2}^{2}}{\lambda_{0}} .
\end{aligned}
$$

The designed VOA beam expansion setup of Fig. 1(a) uses $d_{1}=f_{1}$ and $d_{2}=f_{2}$. Applying these conditions to Eqs. (2a) and (2b) gives

$$
\begin{aligned}
& \frac{\omega_{2}^{2}}{\omega_{1}^{2}}=\frac{f_{1}^{2}}{z_{01}^{2}}=f_{1}^{2} \times \frac{\lambda^{2}}{\pi^{2} \omega_{1}^{4}} \Rightarrow \omega_{2}^{2}=\frac{f_{1}^{2} \lambda^{2}}{\pi^{2} \omega_{1}^{2}}, \\
& \frac{\omega_{3}^{2}}{\omega_{2}^{2}}=\frac{f_{2}^{2}}{z_{02}^{2}}=f_{2}^{2} \times \frac{\lambda^{2}}{\pi^{2} \omega_{2}^{4}} \Rightarrow \omega_{3}^{2}=\frac{f_{2}^{2} \lambda^{2}}{\pi^{2} \omega_{2}^{2}} .
\end{aligned}
$$

Using the value of $\omega_{2}^{2}$ from Eq. (3a) in Eq. (3b) gives

$$
\omega_{3}^{2}=\frac{f_{2}^{2} \lambda^{2}}{\pi^{2}} \times \frac{\pi^{2} \omega_{1}^{2}}{f_{1}^{2} \lambda^{2}} \Rightarrow \frac{\omega_{3}}{\omega_{1}}=\frac{f_{2}}{f_{1}} .
$$

Thus as expected, the foci $f_{1}$ and $f_{2}$ of the lenses $S_{1}$ and $S_{2}$ provide the required magnification factor for the GRIN lens fed Gaussian beam profile. For super-resolution VOA design, $f_{2} / f_{1}$ ratio is maximized to efficiently use the large space bandwidth product of the DMD ${ }^{\mathrm{TM}}$.

\section{EXPERIMENTAL DEMONSTRATION}

The demonstrated VOA shown in Fig. 1 and setup in the laboratory takes advantage of the large micromirror count of the TI tilt-mode $\mathrm{DMD}^{\mathrm{TM}}$ chip designed for infrared $C$ band (1530-1565 nm) operations. This infrared chip has 786432 micromirrors and is designed with $13.8 \mu \mathrm{m}$ square micromirrors operating in bistable $\pm 9.2^{\circ}$ tilt angles. The specified chip PDL is $<0.02 \mathrm{~dB}$ with an individual $15 \mu$ s micromirror switching speed and a $1 \mathrm{~ms}$ total chip setting time through a high speed serial computer interface. The chip optical loss is measured to be $1.9 \mathrm{~dB}$ and includes diffraction loss, pixel fill factor loss, aluminum mirror reflectivity, and hermetically sealed window Fresnel loss. ${ }^{15}$ The three-port circulator $C$ used has a total loss of $1.39 \mathrm{~dB}$ with port 1-to-2 and port 2-to-3 losses of 0.62 and $0.77 \mathrm{~dB}$, respectively. The fiber GRIN lens loss is approximately $0.16 \mathrm{~dB}$. The two planoconvex lenses $S_{1}$ and $S_{2}$ included in the system have infrared antireflection coatings and have a $2 \%$ loss per surface. Thus, for the laboratory reflection based VOA system, total bulk lens induced loss is $0.35 \mathrm{~dB}$. Adding the mentioned component losses, the optical insertion loss for the attenuator is $1.39+0.16+0.35+1.9=3.8 \mathrm{~dB}$. The fiber collimator used has a half self-imaging distance $d_{L}=6 \mathrm{~cm}$. A beam magnification of four is achieved using $S_{1}$ and $S_{2}$ lenses with $f_{1}=5 \mathrm{~cm}$ and $f_{2}=20 \mathrm{~cm}$, respectively. The Gaussian beam at a distance $d_{L}$ from the GRIN lens exhibits a minimum $1 / e^{2}$ beam waist radius $\omega_{1}$ of $0.22 \mathrm{~mm}$ or a null-to-null beam diameter of approximately $3.9 \omega_{1}=0.858 \mathrm{~mm}$. The active area of the $\mathrm{DMD}^{\mathrm{TM}}$ over which the state of micromirrors can be altered is $4.05 \mathrm{~mm} \times 10.92 \mathrm{~mm}$.

As a first experimental setup, the micromirrors on the surface of DMD ${ }^{\mathrm{TM}}$ are accessed column-wise, i.e., along the $x$ direction [see Fig. 1(b)]. Note that the $45^{\circ}$ in-plane rotated orientation of the DMD ${ }^{\mathrm{TM}}$ chip makes the width of the effective column to be the diagonal length of the single micromirror or $13.8 \mu \mathrm{m} / \cos \left(45^{\circ}\right)=19.5 \mu \mathrm{m}$. The present micromirror controls addresses micromirror columns and rows that are oriented at a $45^{\circ}$ angle to the DMD ${ }^{\mathrm{TM}}$ chip frame; hence the $\mathrm{DMD}^{\mathrm{TM}}$ was rotated by $45^{\circ}$. The null-to-null diameter of the expanded Gaussian beam present on the DMD ${ }^{\mathrm{TM}}$ is approximated to be $3.9 \omega_{3}=3.9 \times 4 \times 0.22=3.432 \mathrm{~mm}$ where $\omega_{3}$ $=4 \omega_{1}$. Comparing this $3.432 \mathrm{~mm}$ null-to-null diameter of the 


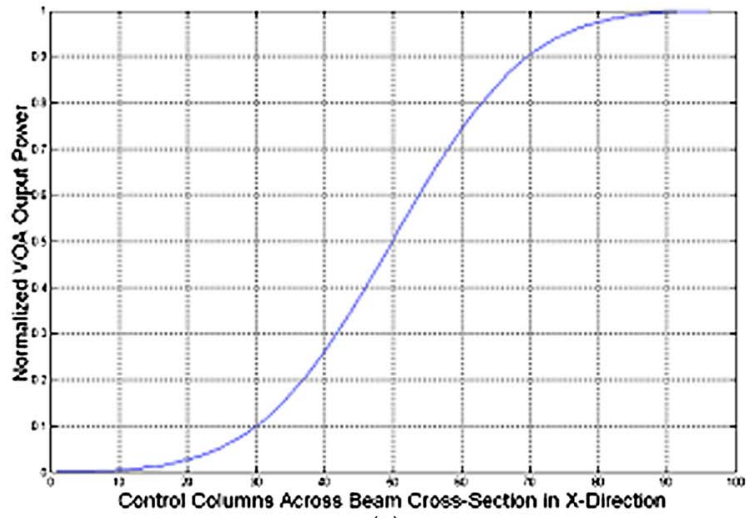

(a)

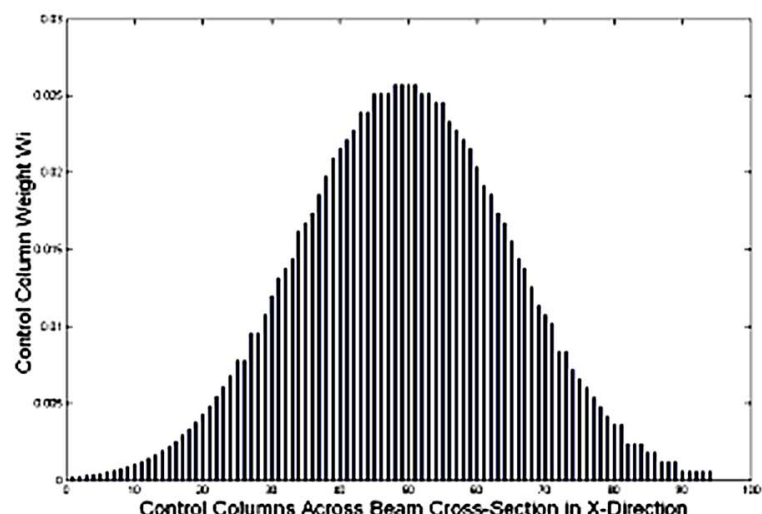

(b)

FIG. 2. (Color online) DMD ${ }^{\mathrm{TM}}$ based super-resolution VOA experimental implementation using the sliding knife edge method. (a) Plot showing the cumulative power obtained as the knife edge on $\mathrm{DMD}^{\mathrm{TM}}$ slides in the $x$ direction, (b) normalized weights for $\mathrm{DMD}^{\mathrm{TM}}$ columns obtained from part (a) data.

expanded beam to the width $w_{\text {col }}=19.5 \mu \mathrm{m}$ of the single effective column gives approximately 176 attenuation control columns within the beam area present on the chip.

Figure 1(a) input SMF is connected to a fiber coupled $1550 \mathrm{~nm}$ laser with stable power control. The output SMF is connected to a precision optical power meter to take VOA attenuation measurements. Initially, all $N$ columns on the $\mathrm{DMD}^{\mathrm{TM}}$ are switched to OFF state, i.e., tilting the micromirrors away from the coupling fiber GRIN lens leading to VOA maximum attenuation. In order to have retroreflective alignment, i.e., light out of the fiber couples back into the fiber, all $\mathrm{DMD}^{\mathrm{TM}}$ micromirrors are set to $\mathrm{ON}$ state or the $+9.2^{\circ}$ setting. Hence, the $\mathrm{DMD}^{\mathrm{TM}}$ chip plane has an overall $9.2^{\circ}$ tilt with respect to the retroreflective beam direction (Fig. 1). Then gradually the columns are switched to the ON state where micromirrors retroreflect light back to the GRIN lens. As the number $i$ of $\mathrm{ON}$-state column increases, more power is coupled back into the fiber lens. This column control process effectively implements a knife edge that gradually slides across the light beam. The results obtained from this experiment are shown in Fig. 2 and give an expected normalized error function profile [Fig. 2(a)] that is differentiated to give a Gaussian function [Fig. 2(b)] that represents the incident optical power variation on the $\mathrm{DMD}^{\mathrm{TM}}$ plane along the direction of the column motion. By normalizing this Gaussian

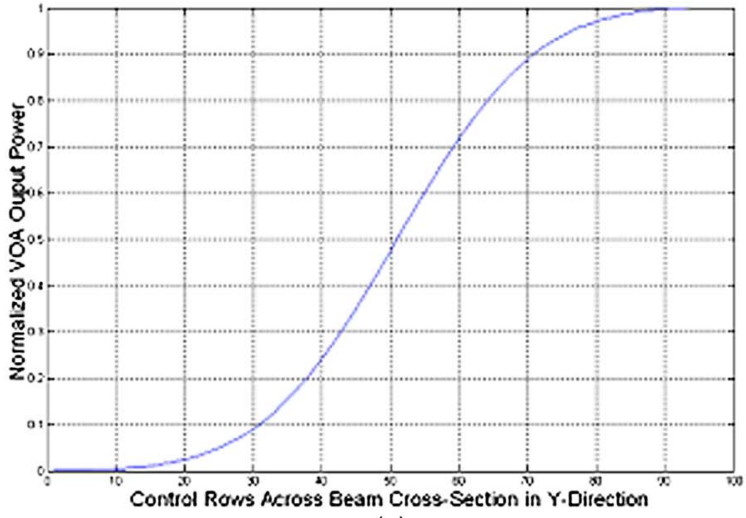

(a)

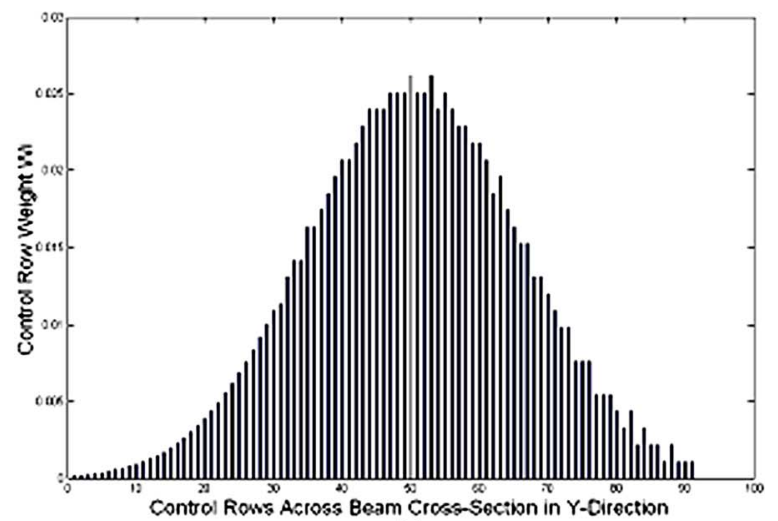

(b)

FIG. 3. (Color online) (a) Plot showing the cumulative power obtained as the knife edge on $\mathrm{DMD}^{\mathrm{TM}}$ slides in the $y$ direction, and (b) normalized weights for $\mathrm{DMD}^{\mathrm{TM}}$ rows obtained from part (a) data.

one dimensional beam profile data, the power attenuation weighting $W_{i}$ response of the $i$ th control column for VOA implementation is given by

$$
W_{i}=\frac{P_{i}-P_{i-1}}{P_{N}},
$$

where $i=1,2 \ldots N$ ( $N=$ total number of columns on $\left.\mathrm{DMD}^{\mathrm{TM}}\right)$. $P_{i}$ is the power for $i$ columns in ON state while $P_{i-1}$ is the power when $(i-1)$ columns are in the ON state. $P_{N}$ is the power when all $N$ control columns on the $\mathrm{DMD}^{\mathrm{TM}}$ are in the $\mathrm{ON}$ state. The calculated column weights to control the VOA are shown in Fig. 2(b) indicating the ratio of the total power controlled by each control column. Note that as expected, the sum of all weights, i.e., $\Sigma_{i=1}^{N} W_{i} \rightarrow 1$. Figures $3(\mathrm{a})$ and $3(\mathrm{~b})$ show similar results when the knife edge is performed in the orthogonal $y$ direction, i.e., by taking rows of micromirrors as the spatial samplers instead of columns. Note that Figs. 2 and 3 data are based on using $N=94$ control columns and $N=91$ control rows as additional column or row usage produces optical power change that falls into the detector noise floor.

The earlier experiment demonstrates the knife-edge method to get simple attenuation control via the proposed VOA. The next experiment involves digital micromirror control over the beam in two dimensions leading to the desired super-resolution VOA. The knife-edge attenuation control mechanism for the VOA demonstrated the use of an effective 
line spatial sampler implemented with at most two adjacent micromirrors making the column width [see Fig. 1(b)]. To realize the super-resolution VOA, a 2-by-2 micromirror group is used as a point spatial sampler. Thus manipulating the state of these effective point spatial samplers distributed in the $2 \mathrm{D}$ area of the $\mathrm{DMD}^{\mathrm{TM}}$ allows super-resolution or finer attenuation control. In the limit, all the individual micromirrors on the $\mathrm{DMD}^{\mathrm{TM}}$ can be used for attenuation control, and in this case an individual micromirror is the effective point spatial sampler. In practice, the limited sensitivity of the VOA characterization Newport Optical Power Meter (model 1830-C) used in the experiment only allows the use of the bigger $2 \times 2$ micromirror spatial sampler so as to obtain readings higher than the detector noise floor. Taking beam waists to be $\omega_{3 x}$ and $\omega_{3 y}$ along $x$ and $y$ dimensions, the total number $N$ of spatial point samplers available for VOA attenuation controls is given by

$$
N=N_{\text {row }} N_{\text {col }}
$$

where $N_{\text {row }}=3.9 \omega_{3 y} / w_{\text {row }}$ and $N_{\text {col }}=3.9 \omega_{3 x} / w_{\text {col }}$. Here, as described for the first experiment, $w_{\mathrm{col}}$ is the width of effective column, whereas, $w_{\text {row }}$ is the width of the effective row for the knife edge performed in the vertical direction [see Fig. $1(\mathrm{~b})] ; w_{\text {row }}=w_{\text {col }}$. Experimentally, the number of spatial samplers $N_{\text {col }}$ along the $x$ dimension and $N_{\text {row }}$ along the $y$ dimension used for VOA control is found to be approximately 176 and 178, respectively. Hence, for complete VOA control there are $176 \times 178$ spatial samplers whose binary on/off states can be used to provide dynamic attenuation. The profile of the laser beam exiting the GRIN lens can be approximated as a 2D Gaussian. Since, a 2D Gaussian function can be written as two one-dimensional (1D) Gaussian orthogonal-coordinates functions, therefore, the normalized 2D weights $W_{i j}$ for the spatial samplers are obtained by the product of the 1D Gaussian weights, i.e., $W_{i}$ and $W_{j}$. In other words,

$$
W_{i j}=W_{i} \times W_{j},
$$

where $W_{i}=\left(P_{i}-P_{i-1}\right) / P_{N_{x}} ; i=1,2 \ldots N_{x} ; N_{x}$ is total number of spatial samplers in the $x$ direction, $W_{j}=\left(P_{j}-P_{j-1}\right) / P_{N_{y}} ; j$ $=1,2 \ldots N_{y} ; N_{y}$ is total number of spatial samplers in the $y$ direction. $P_{N_{x}}$ is the power when all $N$ control columns along the $x$ direction on the $\mathrm{DMD}^{\mathrm{TM}}$ are in the $\mathrm{ON}$ state and $P_{N_{y}}$ is the power when all $N$ control rows along the $y$ direction on the $\mathrm{DMD}^{\mathrm{TM}}$ are in the $\mathrm{ON}$ state. Because the beam profile of the laser beam exiting the GRIN lens has been approximated as a Gaussian, ${ }^{17}$ the weights of the individual spatial samplers, i.e., each $2 \times 2$ micromirror group lying across the beam will also show a Gaussian distribution as shown in Fig. 4. The weights of the $2 \times 2$ micromirror based spatial samplers decrease as we move out radially on the $\mathrm{DMD}^{\mathrm{TM}}$ when compared to the spatial samplers lying towards the center of the beam. At the central zone of the beam, spatial samplers exhibit highest weight and lowest resolution steps. On the other hand, the edges of the beam exhibit spatial samplers on the $\mathrm{DMD}^{\mathrm{TM}}$ with smallest weights and hence highest resolution and VOA beam power control.

The dynamic range of the proposed VOA is tested by observing the difference between the lowest attenuation set-

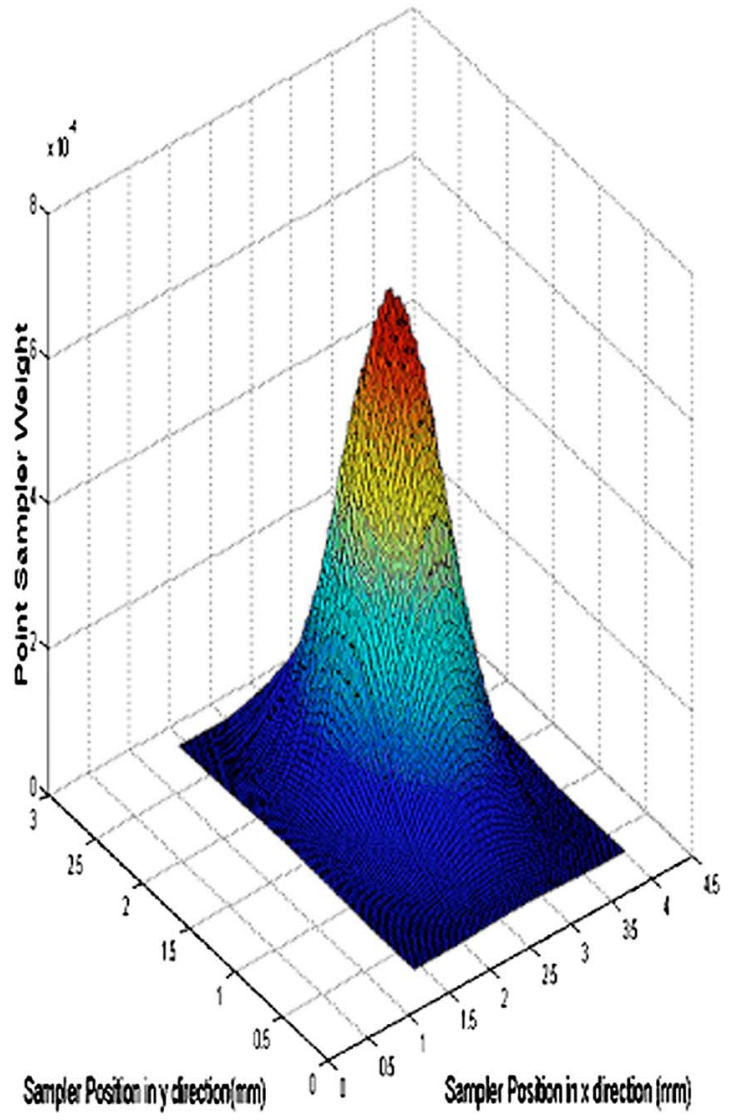

FIG. 4. (Color online) Plot showing computed VOA control weights in 2D for the spatial samplers in the DMD ${ }^{\mathrm{TM}}$. Plot is generated using experimental data shown in Figs. 2 and 3.

ting achieved with all samplers set in their $\mathrm{ON}$ or retroreflective positions versus the maximum attenuation achieved by setting all samplers in their OFF or beam deflection settings. This VOA dynamic range is measured to be $41.5 \mathrm{~dB}$. The circulator and the $\mathrm{DMD}^{\mathrm{TM}}$ chip PDL are specified to be 0.07 and $0.02 \mathrm{~dB}$, respectively, with a successfully tested $<0.07 \mathrm{~dB}$ PDL for the VOA.

\section{VOA SUPER-RESOLUTION}

The largest attenuation change step is obtained using the spatial sampler $2 \times 2$ micromirror set located at the center of the beam giving an attenuation change value of $2.9 \mathrm{mdB}$. The lowest measured VOA step is obtained by using a spatial sampler located at the $1 / e^{2}$ Gaussian beam waist region, giving a VOA attenuation change of $0.0517 \mathrm{mdB}$. Note that there are other lower VOA attenuation control steps produced using the spatial samplers located near the edge of the beam, i.e., beyond $1 / e^{2}$ Gaussian beam waist region but they fall into the noise floor of the present photometer. Figure 5 shows a plot of the demonstrated VOA resolution in decibels $(\mathrm{dB})$ provided by all individual spatial samplers engaged on the $\mathrm{DMD}^{\mathrm{TM}}$. The VOA resolution in $\mathrm{dB}$ is given by the relation 


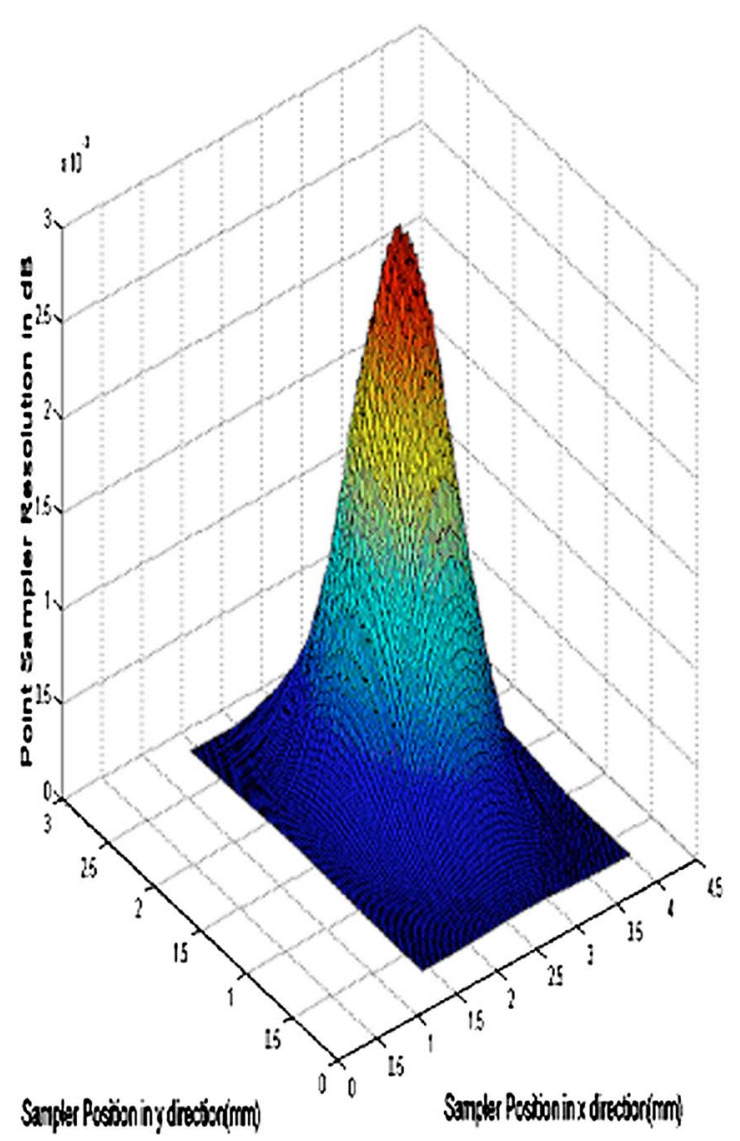

FIG. 5. (Color online) Plot showing the resolution of individual spatial samplers in the DMD ${ }^{\mathrm{TM}}$ that are used for VOA super-resolution controls. Equation (8) is used to generate this data.

$$
R_{i j}=10 \log \left(\frac{1}{1-W_{i j}}\right) \text {. }
$$

Due to the inherent varying nature of attenuation resolution in the proposed VOA instrument, one can define three regions of varying resolution (see Fig. 6). This is done by using two cutoff normalized weight levels $L_{1}$ and $L_{2}$ for the spatial samplers and defining three regions through these levels. An example of defined levels is shown in Fig. 6 using normalized weight cutoffs of $L_{1}=0.225$ and $L_{2}=0.425$. The attenuator can make intelligent use of these three available resolution regions to arrive at a given VOA attenuation setting with extraordinarily high resolution. The number of samplers in each of the three regions is obtained by counting the samplers that satisfy the cutoff normalized weight level conditions shown in Fig. 6. This is done by the following matrix operations. As a first step, all matrices required for the VOA super-resolution operations are defined. The normalized weight matrix $\mathbf{W}$ is defined as

$$
\mathbf{W}=\left[W_{i j}\right]_{N_{\text {row }} \times N_{\text {col }}},
$$

where $i=1,2 \ldots N_{\text {col }}$ and $j=1,2 \ldots N_{\text {row }}$. As shown next by the relations in Eq. (10). Matrices $\mathbf{M}_{1}, \mathbf{M}_{2}$, and $\mathbf{M}_{3}$ are obtained by comparison of all elements of the weight matrix $\mathbf{W}$ with the normalized weight levels $L_{1}$ and $L_{2}$, i.e.,

$$
\begin{aligned}
& \operatorname{if}\left(W_{i j}<L_{1}\right) \\
& M_{1 i j}=1,
\end{aligned}
$$

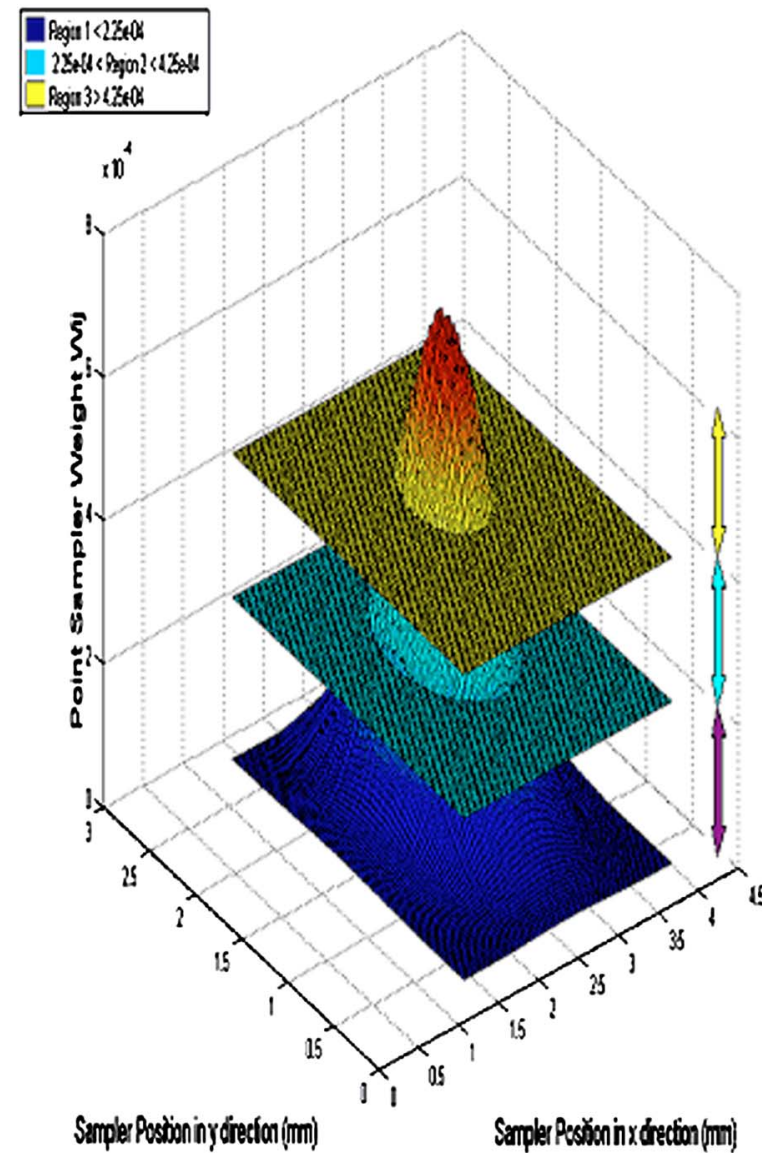

FIG. 6. (Color online) Plot showing the three segmented regions of VOA normalized weights and the threshold levels $L_{1}=0.225$ and $L_{2}=0.425$ separating them. Figure 4 data are used to generate this plot.

else

$M_{1 i j}=0$

$\operatorname{if}\left(L_{1} \leqslant W_{i j}<L_{2}\right)$

$M_{2 i j}=1$,

else

$M_{2 i j}=0$

if $\left(W_{i j} \geqslant L_{2}\right)$

$M_{3 i j}=1$,

else

$M_{3 i j}=0$

for $i=1,2 \ldots N_{\text {col }}$ and $j=1,2 \ldots N_{\text {row }}$. In other words, the matrices $\mathbf{M}_{1}, \mathbf{M}_{2}$, and $\mathbf{M}_{3}$ contain a "1" for a corresponding element weight in $\mathbf{W}$ matrix satisfying the Eq. (10) threshold condition and a "0" for not satisfying the condition.

Next, an operator "Sum" $S[M]$ is defined that adds all the elements of a matrix $M$. As the second processing step, the contents of each of the matrices $\mathbf{M}_{1}, \mathbf{M}_{2}$, and $\mathbf{M}_{3}$ are added by using Sum operator giving 


$$
\begin{aligned}
& N_{1}=S\left[\mathbf{M}_{1}\right], \\
& N_{2}=S\left[\mathbf{M}_{2}\right], \\
& N_{3}=S\left[\mathbf{M}_{3}\right] .
\end{aligned}
$$

Hence, $N_{1}, N_{2}$, and $N_{3}$ are the number of samplers in the three defined regions.

As the third step, another operator is defined as "dotmultiplication" $(\diamond)$ that multiplies the corresponding elements of two matrices if they have the same order. The Sum operator of the dot-multiplication of matrices $\mathbf{M}_{1}, \mathbf{M}_{2}$, and $\mathbf{M}_{3}$ with $\mathbf{W}_{i j}$ gives the ratio of power falling in each of the defined regions and thus the power percentages can be written as

$$
\begin{aligned}
& P_{1}=S\left[\mathbf{W}_{i j} \diamond \mathbf{M}_{1 i j}\right] \times 100, \\
& P_{2}=S\left[\mathbf{W}_{i j} \diamond \mathbf{M}_{2 i j}\right] \times 100, \\
& P_{3}=S\left[\mathbf{W}_{i j} \diamond \mathbf{M}_{3 i j}\right] \times 100 .
\end{aligned}
$$

With weight matrix $\mathbf{W}$ containing weights as shown in Fig. 4 and normalized weight cutoffs of $L_{1}=0.225$ and $L_{2}=0.425$ as mentioned earlier, the matrices $\mathbf{M}_{1}, \mathbf{M}_{2}$, and $\mathbf{M}_{3}$ are calculated according to Eqs. (10a)-(10c). Using Eq. (11), $N_{1}, N_{2}$, and $N_{3}$ equal 7097, 995, and 648, respectively. Similarly, using Eq. (12) gives $P_{1}, P_{2}$, and $P_{3}$ values of $33.77 \%$, $31.51 \%$, and $34.71 \%$, respectively.

The weights of the $2 \times 2$ micromirror based spatial samplers lying across the beam cross section follow the given incident beam profile strength on the DMD ${ }^{\mathrm{TM}}$. Since the spatial samplers in the defined region 3 lie in the center of the beam, they carry more weight and account for a larger percentage of the beam power. Specifically for the design shown in Fig. 6, region 3 controls $P_{3}=34.71 \%$ of the total beam power falling on the $\mathrm{DMD}^{\mathrm{TM}}$ and has $N_{3}=648$ spatial samplers. The average resolution for the samplers in this region is given by $R_{3 \text { ave }}=-10 \log \left(P_{3} / 100\right) / N_{3}$ $=-10 \log (34.71 / 100) / 648=7.092 \mathrm{mdB}$. Earlier we defined a spatial sampler as a group of $2 \times 2$ micromirrors. This sampler definition was shown to keep the power readings at the edge of the beam to be above the measuring detector noise floor. Since, region 3 does not include the beam edge and has sufficient power within each sampler; the $2 \times 2$ micromirrors lying within each sampler can be manipulated to obtain 3
$(4-1=3)$ more steps in the overall VOA attenuation control leading to an average VOA in the fine control range of a resolution of $1.773 \mathrm{mdB}$. Region 2 handles $P_{2}=31.51 \%$ of the total power with a total of $N_{2}=995$ spatial samplers. This gives an average resolution of $R_{2 \text { ave }}=-10 \log \left(P_{2} / 100\right) / N_{2}$ $=5.041 \mathrm{mdB}$. Like region 3 , micromirrors within the defined spatial sampler can also be altered for region 2 to introduce more steps for the attenuator. Finally, region 1 handles the power along the outermost region of the beam where it controls $P_{1}=33.77 \%$ of the total power. Since there is less power in the outer region of the beam, this percentage is spread over a large number of samplers, i.e., $N_{1}=7097$. This gives an extremely high average VOA resolution for this region 1 to be $R_{1 \text { ave }}=-10 \log \left(P_{1} / 100\right) / N_{1}=0.664 \mathrm{mdB}$.

Thus, for operational purposes the VOA can be divided into three resolution zones and micromirrors in these zones can be further manipulated to deliver the desired high resolution attenuator setting. The module is suited for VOA test instrument applications. Future work relates to optimization of the beam shaping optics and micromirror controls to enable full use of the DMD ${ }^{\mathrm{TM}}$ space bandwidth product.

${ }^{1}$ S. Masuda, Appl. Opt. 19, 2435 (1980).

${ }^{2}$ J. E. Ford, J. A. Walker, D. S. Greywall, and K. W. Goossen, J. Lightwave Technol. 16, 1663 (1998).

${ }^{3}$ C. Marxer, P. Griss, N. F. de Rooij, IEEE Photonics Technol. Lett. 11, 233 (1999).

${ }^{4}$ E. G. Hanson, Appl. Opt. 21, 1342 (1982).

${ }^{5}$ N. A. Riza and N. Madamopoulos, Appl. Opt. 38, 2309 (1999).

${ }^{6}$ K. Hirabayashi, M. Wada, and C. Amano, Appl. Opt. 40, 3509 (2001).

${ }^{7}$ T. Loukina, R. Chevallier, J. L. de Bougrenet de la Tocnaye, and M. Barge, J. Lightwave Technol. 21, 2067 (2003).

${ }^{8}$ P. Chanclou, B. Vinouze, M. Roy, C. Cornu, and H. Ramanitra, J. Lightwave Technol. 21, 3471 (2003).

${ }^{9}$ S. Wada, S. Abe, Y. Ota, B. Reichman, T. Imura, and C. A. Daza, Optical Fiber Communication Conference (OFC), 2002, p. 324.

${ }^{10} \mathrm{~S}$. Sumriddetchkajorn and K. Chaitavon, IEEE Photonics Technol. Lett. 16, 1507 (2004).

${ }^{11}$ M. I. Braiwish, B. L. Bachim, and T. K. Gaylord, Appl. Opt. 43, 1789 (2004).

${ }^{12}$ M. J. Mughal and N. A. Riza, IEEE Photonics Technol. Lett. 14, 510 (2002).

${ }^{13}$ N. A. Riza, US Patent No. 6,222,954 (2001).

${ }^{14}$ N. A. Riza and S. Sumriddetchkajorn, Opt. Lett. 24, 282 (1999).

${ }^{15}$ N. A. Riza and M. J. Mughal, Opt. Eng. (Bellingham) 11, 1559 (2003).

${ }^{16}$ S. Sumriddetchkajorn and N. A. Riza, Opt. Commun. 205, 77 (2002).

${ }^{17}$ M. van Buren and N. A. Riza, Appl. Opt. 42, 550 (2003).

${ }^{18}$ A. Yariv and P. Yeh, Optical Waves in Crystals (Addison-Wesley, San Francisco, CA, 1984). 